\title{
Percutaneous Mitral Valve Repair of Mitral Valve Prolapse using Mitraclip
}

\author{
Francis Carl L. Catalan ${ }^{1 *}$, Pierre Albert A. Alora ${ }^{1}$, Mark Gavin M. Garcia ${ }^{2}$, Ariel Miranda ${ }^{3}$, Melissa Co-Sia $^{4}$ and Timothy Dy $^{4}$ \\ ${ }^{1}$ Cardiology Research Fellow, Section of Invasive Cardiology, Cardinal Santos Medical Center, Philippines \\ ${ }^{2}$ Cardiology Fellow, Section of Adult Cardiology, Cardinal Santos Medical Center, Philippines \\ ${ }^{3}$ Active Consultant and Head, Section of Invasive Cardiology, Cardinal Santos Medical Center, Philippines \\ ${ }^{4}$ Active Consultant, Section of Invasive Cardiology, Cardinal Santos Medical Center, Philippines
}

\begin{abstract}
Background: Chronic mitral regurgitation can be divided into primary and secondary. The most common cause of chronic primary mitral regurgitation in developed countries is mitral valve prolapse. Many patients remain asymptomatic for quite some time, although its presence has an impact on the prognosis that is why it is mandatory to be address appropriately. In patients with chronic symptomatic, severe mitral regurgitation from degenerative cause, surgical mitral valve repair remains the gold standard. However, percutaneous mitral valve repair has been in progress in different countries and it has shown propitious result. Mitraclip restores leaflet coaptation by approximating the edges of the regurgitant mitral valve leaflets under transesophageal echocardiography and fluoroscopic guidance.

Case: This is a case of a 61year old male, hypertensive, who came in for routine checkup prior to endoscopy which showed severe mitral regurgitation from posterior mitral valve prolapse three years ago. He was given the option to undergo surgical versus transcutaneous repair of the mitral regurgitation but opted to be treated medically. Four months prior to consult patient underwent emergency ventriculo-peritoneal shunting and coil embolization secondary to left posterior cerebral artery aneurysm. He was discharged improved with no residual deficit. Afterwards, the patient reconsidered the choice of mitraclip.

Diagnostics and therapeutics: Work-up done to the patient revealed moderate coronary artery disease of the ramus intermedius and severe mitral regurgitation secondary to posterior mitral valve prolapse for which he underwent percutaneous mitral clip.

Conclusion: The successful performance of mitraclip in our institution offers our future patients an alternative to surgical repair or replacement. It is comparable to surgery in terms of reducing the left ventricular volume, dimension and mitral gradient.
\end{abstract}

\section{Background}

Mitral regurgitation or insufficiency is a common heart valve problem and treatment will depend on the severity and the presence of symptoms. Mild to moderate mitral regurgitation usually does not require treatment whereas the gold standard for chronic, severe MR is surgical replacement or repair of valve [1,2]. Chronic mitral regurgitation can be divided into primary and secondary. The most common cause of chronic primary mitral regurgitation in developed countries is mitral valve prolapse [3]. Many patients remain asymptomatic, but despite this, the severity of mitral regurgitation has an impact on the prognosis of mitral valve prolapse. It usually has frequent and serious complications, including mitral regurgitation requiring surgery, stroke, atrial fibrillation, and heart failure [1-3]. Continued follow-up is needed and it is mandatory that symptoms and changes on the valve defect be addressed accordingly and appropriately. In patients with chronic symptomatic, severe mitral regurgitation from degenerative cause, surgical mitral valve repair remains the treatment of choice [4]. Search for a safer solution for high-risk individuals in whom surgery poses a great threat drove the use of the MitraClip procedure [5]. MitraClip produced lower rates of hospital mortality and adverse events according to ACCESS-EU study [6] Mitraclip approximates the edges of the regurgitant mitral valve restoring leaflet coaptation. This is being done under transesophageal echocardiography and fluoroscopic guidance [7]. Worldwide, there is increasing use of the said procedure. Several clinical registries in the USA and Europe established its high procedural success rates, safety and durability [8,9]. In the Philippines, there were only two previous attempts for MitraClip Procedure and only one was successful. This paper aims to present the $3^{\text {rd }}$ attempt for Mitra Clip Procedure here in the Philippines and to discuss the techniques done which possibly contributed to a successful outcome.

\section{Case report}

A 61-year old male came in our institution for routine checkup. Patient claimed to be asymptomatic, physically active without limitations of his daily activities. He consulted with a private physician and requested to have endoscopy. On physical examination, there was a presence of murmur hence $2 \mathrm{~d}$-echocardiogram was requested and revealed severe mitral regurgitation secondary to a posterior mitral valve prolapse. He was referred to a cardiologist for further management. $\mathrm{He}$ was advised to undergo repair via surgery or percutaneous procedure but opted medical therapy initially. On the interim, patient developed

Correspondence to: Francis Carl L. Catalan, Cardiology Research Fellow, Section of Invasive Cardiology, Cardinal Santos Medical Center, Philippines; E-mail: franciscarlmd@gmail.com

Key words: Mitral Valle Prolapse, MitraClip

Received: February 28, 2017; Accepted: March 21, 2017; Published: March 24, 2017 
severe headache, and was rushed to a nearby hospital. Blood pressure was elevated at 178/104 with progressive decrease in sensorium. Cranial CT scan revealed aneurysm of the posterior cerebral artery with increase in intracerebral pressure. He underwent emergency ventriculostomy and was transferred to our institution for coiling of the left posterior cerebral artery aneurysm. He was discharged well and after one month was noted to be ambulatory with no neurologic deficit.

He had close follow up at outpatient for monitoring. The option of undergoing trans-catheter repair of the mitral regurgitation was contemplated. After four months of follow up, he was admitted for the Mitraclip procedure. On physical examination, he was conscious, coherent and ambulatory, with a BMI of $21.5 \mathrm{~kg} / \mathrm{m}^{2}$. He had stable vital signs of 100/60, 75, 18 with a JVP of $5 \mathrm{cmH}_{2} 0$. He had dynamic precordium, AB at the $6^{\text {th }}$ LICS, MCL (+) LV heave, (+) thrill, normal S1 and S2, (+) 4/6 holosystolic murmur area best heard on apical area accentuated by standing, palpable P2, with no Carvallo's sign. Rest of the physical and neurologic examination was normal. His maintenance medications were Perindopril, Amlodipine, Atorvastatin and Clopidogrel.

\section{Diagnostics}

Prior to the procedure, diagnostic examinations were requested. His 12-L ECG was sinus rhythm with normal axis, left atrial enlargement and left ventricular hypertrophy (Figure 1). In Figure 2, his chest radiogram showed an enlarged heart with left ventricular (LV)

Table 1. Anatomical Selection Criteria for the MitraClip device adapted from Maisano et al. [10].

\begin{tabular}{|l|}
\hline Anatomical Selection Criteria for the MitraClip Device \\
Recommended anatomical criteria (from the EVEREST trial) \\
MR originates from the A2-P2 area \\
Coaptation length $>2 \mathrm{~mm}$ \\
Coaptation depth $<11 \mathrm{~mm}$ \\
Flail gap $<10 \mathrm{~mm}$ \\
Flail width $<15 \mathrm{~mm}$ \\
Mitral valve orifice area $>4 \mathrm{~cm}^{2}$ \\
Additional Criteria for Caution \\
\hline Short posterior leaflet $(<8 \mathrm{~mm})$ \\
Restricted posterior leaflet prolapse/flail width $>15 \mathrm{~mm}$ \\
Calcification in the grasping area \\
Cleft or subcommissures in the area of the jet \\
\hline
\end{tabular}

prominence. Main pulmonary artery (MPA) was not dilated. Aorta is tortuous. The right hemidiaphragm and costophrenic sulci were intact.

The patient's $2 \mathrm{D}$ echocardiogram showed thickened and flail mitral valve leaflet with severe mitral regurgitation with mitral velocity of 4.84 $\mathrm{m} / \mathrm{s}$ with mitral gradient of $93.8 \mathrm{mmHg}$ (Figure 3-6)

\section{Discussion}

For degenerative mitral regurgitation, surgical mitral valve repair or replacement has been the norm. The standardized procedure is the edge-to-edge technique which was first performed in the 1990s. The procedure allowed for improvement, efficacy and durability of the repair [10]. However, some patients have significant comorbidities and/ or of advanced age hence, are reluctant to undergo surgery. This same technique also allowed for the feasibility of a percutaneous approach [10]. A surgical repair of the mitral valve was not considered by the patient and the family due to his past medical history. The ramifications seen during his previous neurologic event prevented his family from choosing surgery, and opted for the less invasive mitraclip procedure. To determine the success of the MitraClip procedure, it is important that the following should be strictly complied with: (a) precise and stable delivery system, (b) solid and reliable tissue approximation, (c) good visualization of the devise and lastly (d) good visualization of the device considering its repositionability and retrievability [10-12]. Based on clinical data [13] approximation of coaptation with MitraClip has been shown to be more efficient as compared with suture. Aside from the above criteria, the EVEREST trial tabulated the anatomic selection criteria (Table 1) (Figure 7-10)

\section{Percutaneous MVR procedure}

The two-part system of MitraClip consists of the Clip delivery system and the Steerable guide catheter. The device is usually made of a polyester fabric and a metal alloy. The way it function is like doing an Alfieri repair technique wherein it grasps the mitral leaflets causing an end-to-end approximation. The patient was sedated and intubated under general anesthesia. Transvenous access through right femoral was done. Atrial transeptal puncture was performed via transesophageal guidance. Thereafter, the MitraClip was fed through the catheter and directed towards the mitral valve where it is aligned with the maximum jet originating from the regurgitant lesion. The clip

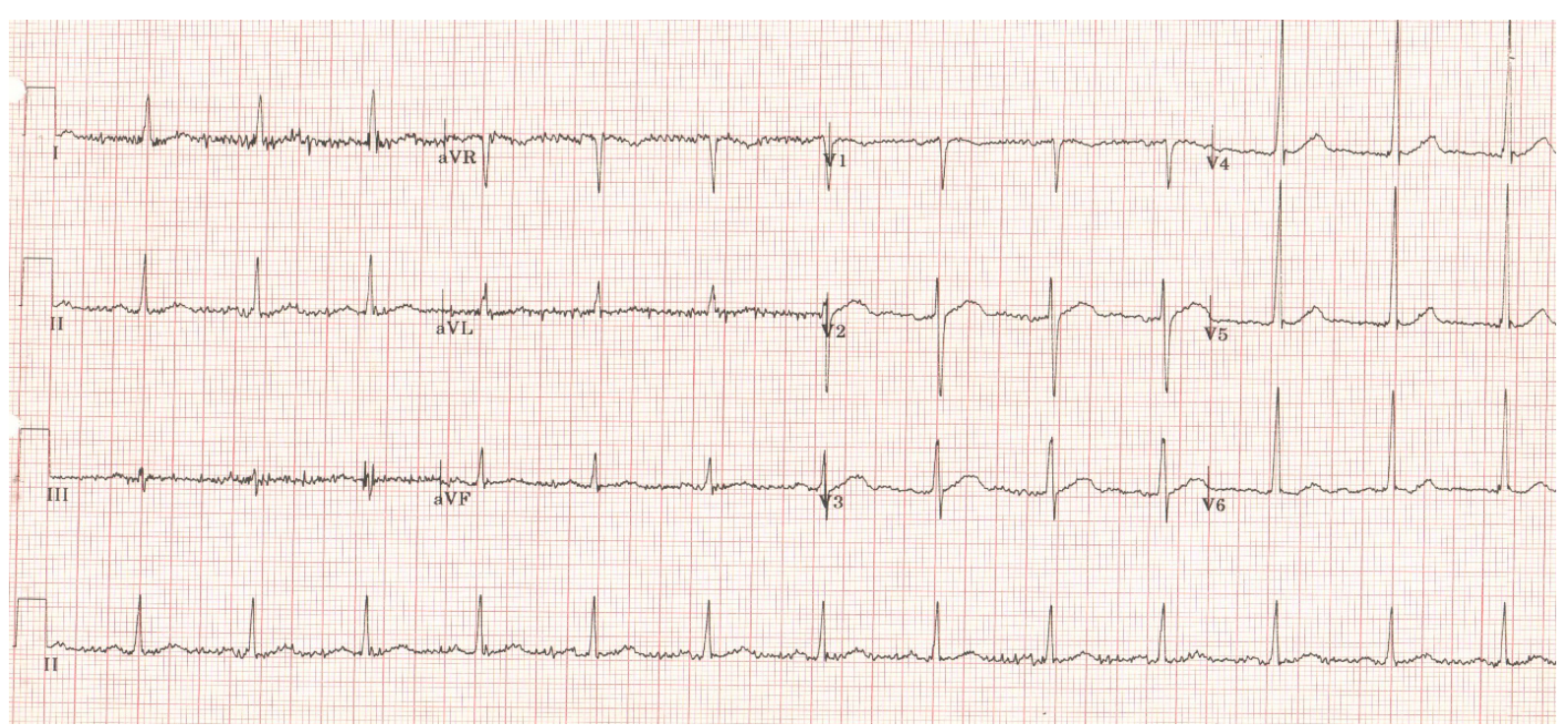

Figure 1. 12-L-ECG showed sinus rhythm, normal axis, left atrial enlargement and left ventricular hypertrophy. 


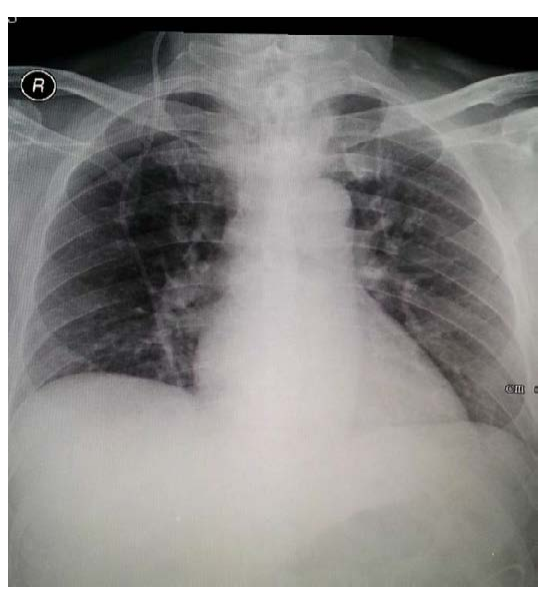

Figure 2. Chest radiogram showed an enlarged heart with ventricle (LV) prominence. Main pulmonary artery (MPA) was not dilated. Aorta is tortuous. Right hemidiaphragm and CPS are intact.

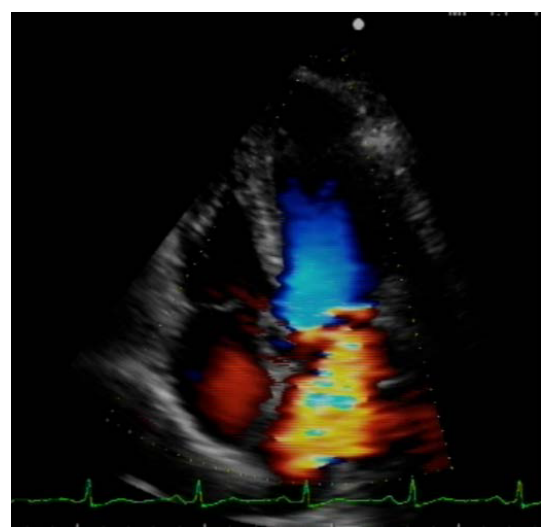

Figure 3. Apical four chamber view showing thickened and flail mitral valve leaflet with severe mitral regurgitation.

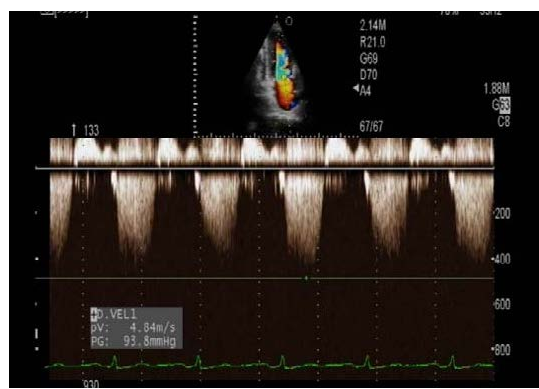

Figure 4. On Apical two chamber view. Mitral velocity of $4.84 \mathrm{~m} / \mathrm{s}$ with a mitral gradient of $93.8 \mathrm{mmHg}$.

was adjusted and positioned several times to allow for the device to have a better grasp of the leaflets. After adequate reduction of regurgitation using multiple echo views, the first clip was deployed approximating the A2P2 segments. Persistent moderate regurgitation necessitated a second clip to be deployed. The second clip was inserted and positioned near the first deployed clip. The thorough views of TEE provided the best position to deploy the second clip reducing the regurgitation remarkably to trivial.

The previously mentioned selection criteria for valves with the best morphology that warrants mitraclip are again the following: mitral valve opening area $>4 \mathrm{~cm}^{2}$, mobile length of the posterior leaflet $\geq 10 \mathrm{~mm}$ and coaptation depth $<11 \mathrm{~mm}$, normal leaflet strength and

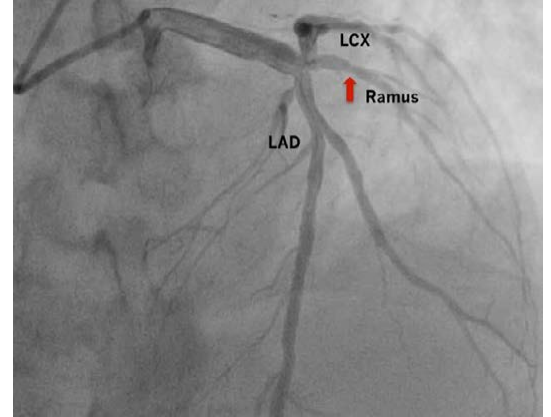

Figure 5. Coronary angiogram showing moderate coronary artery disease of the ramus intermedius.

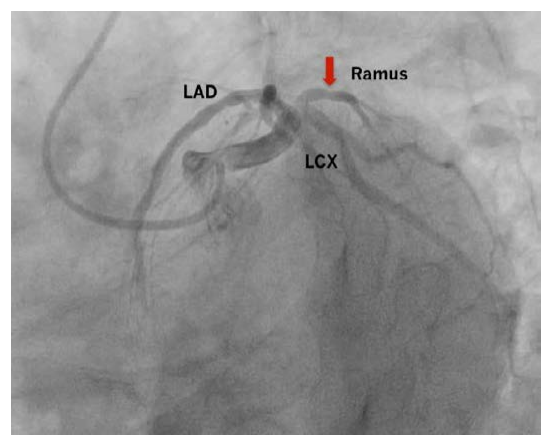

Figure 6. Coronary angiogram showing moderate coronary artery disease of the ramus intermedius.

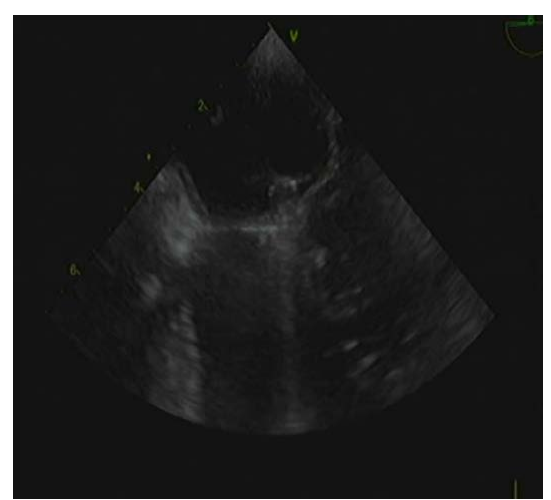

Figure 7. Patient underwent Transcatheter repair of mitral regurgitation via mitraclip of the A2P2 segments. (First Clip deployed).

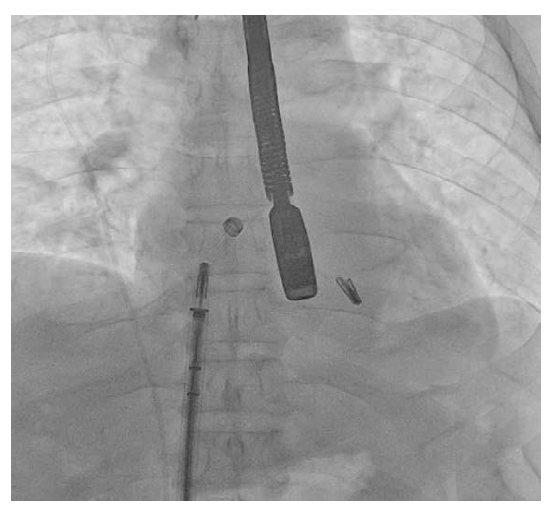

Figure 8. Patient underwent Transcatheter repair of mitral regurgitation via mitraclip of the A2P2 segments. (First Clip deployed).

mobility, flail width $<15 \mathrm{~mm}$ and flail gap $<10 \mathrm{~mm}$, central pathology in segment 2 with no calcification [12]. In our patient, he had a central 


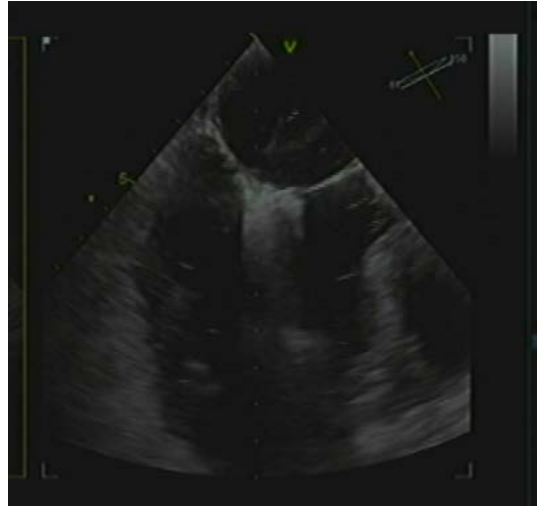

Figure 9. Patient underwent Transcatheter repair of mitral regurgitation via mitraclip of the A2P2 segments. (2 clips deployed).

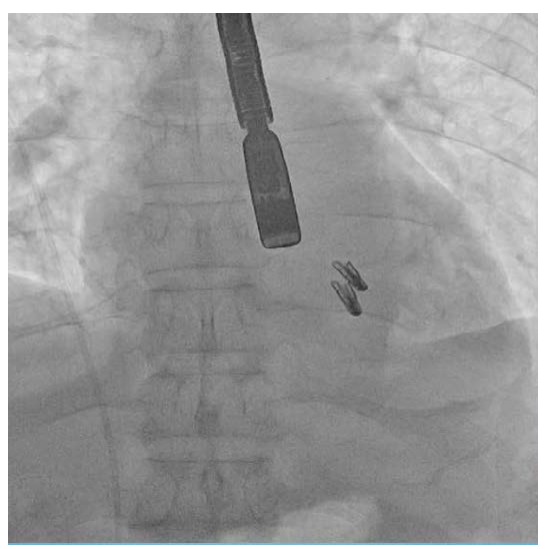

Figure 10. Patient underwent Transcatheter repair of mitral regurgitation via mitraclip of the A2P2 segments. (2 clips deployed)

pathology with no calcification, flail gap of $4.7 \mathrm{~mm}$ and flail width of $4.1 \mathrm{~mm}$. The two mitraclips were placed on the A2P2 segments where it gave a good grip of the leaflets approximating thru the entire cardiac cycle. Here in the Philippines, this is the third attempt for MitraClip procedure and $2^{\text {nd }}$ successful one. This percutaneous repair thru mitraclip was the first case in Cardinal Santos Medical Center. Patient tolerated the procedure with uneventful hospital stay. He was discharged after 3 days. He is currently maintained on clopidogrel, aspirin, atorvastatin, perindopril, amlodipine, carvedilol, mucosta and pantoprazole. Currently, patient is still asymptomatic with no murmur noted and a trivial mitral regurgitation on echocardiogram. According to EVEREST trial, percutaneous repair of the mitral valve has no difference to surgery in the future occurrence of MR or mitral valve intervention between 1 year and 4 year follow up. This translates to be an alternative strategy to mitral surgery.

\section{Incidence of failure and reasons}

Patients treated with MitraClip who failed to satisfy the EVEREST Trial Anatomical Selection Criteria was associated with more procedural failure and shorter durability.

\section{Conclusion}

The successful performance of this percutaneous approach in our institution offers our future patients an option for a safe, advantageous and effective treatment modality for patients who needs mitral valve repair but at the same time is high risk for surgical procedure because of co-morbidities.

\section{Acknowledgment}

The authors thank Dr. Yasmin Lee-Catalan and Mr. Charles Santiago for their continued support and assistance in editing the files and figures.

\section{References}

1. Avierinos JF, Gersh BJ, Melton LJ 3rd, Bailey KR, Shub C, et al. (2002) Natural history of asymptomatic mitral valve prolapse in the community. Circulation 106: 1355-1361. [Crossref]

2. Nishimura RA, Otto CM, Bonow RO, Carabello BA, Erwin JP 3rd, et al. (2014) AHA ACC guidelines for the management of patients with valvular heart disease: a report of the American College of Cardiology/ American Heart Association Task Force on Practice Guidelines. Circulation 129: 2440-2492. [Crossref]

3. Freed LA, Levy D, Levine RA, Larson MG, Evans JC, et al. (1999) Prevalence and clinical outcome of mitral-valve prolapse. N Engl J Med 341: 1-7. [Crossref]

4. Mauri L, Foster E, Glower DD, Apruzzese P, Massaro JM, et al. (2013) 4-year results of a randomized controlled trial of percutaneous repair versus surgery for mitral regurgitation. J Am Coll Cardiol 62: 317-328. [Crossref]

5. Franzen O, Baldus S, Rudolph V, Sven Meyer1, Malgorzata Knap, et al. (2010) Acute outcomes of MitraClip therapy for mitral regurgitation in high-surgical-risk patients: emphasis on adverse valve morphology and severe left ventricular dysfunction. European Heart Journal 31: 1373-1381.

6. Maisano F, Franzen O, Baldus S, Schäfer U, Hausleiter J, et al. (2013) Percutaneous Mitral Valve Interventions in the Real World. Early and 1-Year Results From the ACCESS-EU, A Prospective, Multicenter, Nonrandomized Post-Approval Study of the MitraClip Therapy in Europe. J Am Coll Cardiol 62: 1052-1061. [Crossref]

7. Sürder D, Pedrazzini G, Gaemperli O, Biaggi P, Felix C, et al. (2013) Predictors for efficacy of percutaneous mitral valve repair using the MitraClip system: the results of the MitraSwiss registry. Heart 99: 1034-1040. [Crossref]

8. Gaemperli O, Corti R. (2012) Mitraclip for the treatment of mitral regurgitation Cardiovascular Medicine 15: 276-286.

9. Puls M, Lubos E, Boekstegers P, Bardeleben RSV, Ouarrak T, et al. (2015) One-year outcomes and predictors of mortality after MitraClip therapy in contemporary clinical practice: results from the German transcatheter mitral valve interventions registry. European Heart Journal doi:10.1093.

10. Maisano F, La Canna G, Colombo A, Alfieri O (2011) The evolution from surgery to percutaneous mitral valve interventions: the role of the edge-to-edge technique. $J \mathrm{Am}$ Coll Cardiol 58: 2174-2182. [Crossref]

11. Maisano F, Denti P, Michev I, et al. (2009) Percutaneous mitral valve repair with the edge-to-edge technique. Multimedia Manual of Cardiothoracic Surgery / doi:10.1510/ mmcts.004002.

12. Boekstegers P, Hausleiter J, Baldus S, von Bardeleben RS, Beucher H, et al. (2014) Percutaneous interventional mitral regurgitation treatment using the Mitra-Clip system. Clin Res Cardiol 103: 85-96.

13. Feldman T, Kar S, Elmariah S, Smart SC, Trento A, et al. (2015) 5 year Clinical Outcomes: Percutaneous Repair and Surgery for Mitral Regurgitation. EVEREST II Pivotal Study High Risk Registry. J Am Coll Cardiol 66: 2844-2854.

Copyright: (C2017 Catalan FCL. This is an open-access article distributed under the terms of the Creative Commons Attribution License, which permits unrestricted use, distribution, and reproduction in any medium, provided the original author and source are credited. 